\title{
Gate Control System for New Iraqi License Plate
}

\author{
Mr.Furat Nidhal Tawfeeq ${ }^{1}$, Mrs. Yasmine Mazin Tabra ${ }^{2}$ \\ ${ }^{1}$ Iraqi National Cancer Research Center/ Baghdad University \\ furatnidhal@yahoo.com \\ ${ }^{2}$ College of Information Engineering \\ / Al-Nahrain University \\ yasminetabra@yahoo.com
}

\begin{abstract}
This paper presents an approach to license plate localization and recognition. A proposed method is designed to control the opening of door gate based on the recognition of the license plates number in Iraq. In general the system consists of four stages; Image capturing, License plate cropping, character segmentation and character recognition. In the first stage, the vehicle photo is taken from standard camera placed on the door gate with a specific distance from the front of vehicle to be processed by our system. Then, the detection method searches for the matching of the license plate in the image with a standard plate. The segmentation stage is performed by is using edge detection. Then character recognition, done by comparing with template standard numbers and letters used in the Iraqi plate. The system was implemented using Matlab (R2012a) and shows accurate performance results reached 93.33\%.
\end{abstract}

Keywords: License Plate Recognition; Edge Detection; Template Matching; Character Recognition.

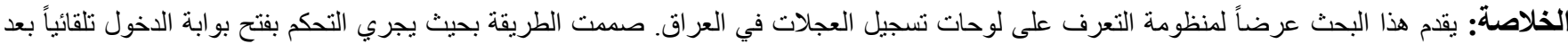

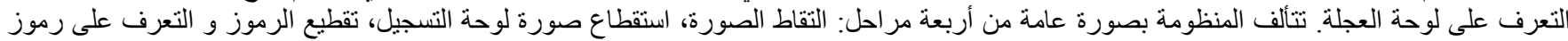

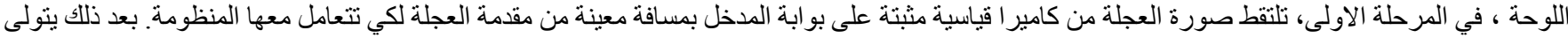

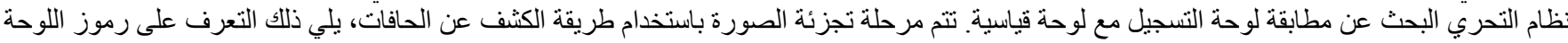

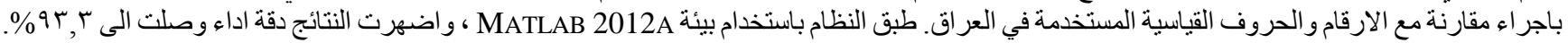

\section{Introduction}

License Plate Recognition (LPR) is an image processing technology used to identify vehicles by their number plates (Wangunyu, 2009).

The first invention of the Automatic Number Plate Recognition (ANPR) was in 1976 at the Police Scientific Development Branch in the UK. Prototype systems were working by 1979 and contracts were let to produce industrial systems, first at EMI Electronics then at Computer Recognition Systems (CRS) in Wokingham and UK.

License Plate Recognition System (LPRS) is a challenging problem in the field of machine vision and automation with various applications including law enforcement, parking lot ticketing systems, automated hands free toll collection, automated vehicle access in secure establishments etc (Sudip Roy, 2007).

The task of recognizing specific object in an image is one of the most difficult topics in the field of computer vision or digital image processing (Kaushik Deb, 2009)

Many methods have been proposed to detect license plates from car images. Most researchers prefer a hybrid detection algorithm in order to make the algorithm more robust and accurate. People usually manage to get some candidate regions based on the features that appear uniquely in number plate regions. Then, combined with some prior knowledge of license plates, regions other than plate region are removed and license plate regions are left, which means license plates are successfully detected (Wenjing Jia, 2004).

License Plate Character Segmentation plays the role to segment character from the located region of license plate, based on license plate location results. The segmentation result of license plate character is the precondition and preparation for character recognition. License plate character recognition is mainly to process, extract character feature and identify the license plate characters segmented. Character recognition is the critical step in the system, which directly determines the performance of the recognition system (Jian-Xia Wang et. al, 2010).

One of the applications of LPR system is the open gate system which will be used in this paper, by identifying all the character and regions in the new Iraqi license plate written by using Indian and English characters (letters and numbers).

\section{New Iraqi License Plates}

This proposed system uses the new Iraqi License Plate to be detected from vehicle picture taken by camera. The Iraqi License plate consists of several parts that are used as unique items in the identification of the plate for extraction. Figure (1) shows a license plate with the regions described below.

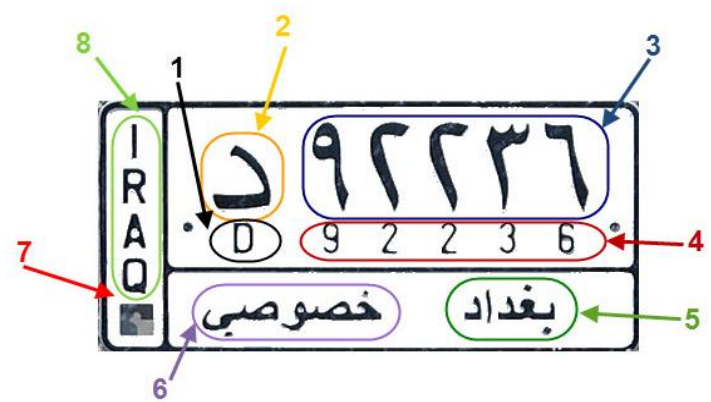

Figure 1: Iraqi License Plate 
Region 1: This region is represented by one digit of English characters.

Region 2: This region is represented by one digit of Indian (Arabic) characters.

Region 3: This region is represented by maximum of five digits of Indian (Arabic) numbers.

Region 4: This region is represented by maximum of five digits of English numbers.

Region 5: This region is an Indian (Arabic) string represents governorate in which the car was registered.

Region 6: This region represents the type of the car (personal, transport, taxi, Governmental). This region is also represented in Indian (Arabic) Strings.

Region 7: This region is colored depending on the car types mentioned in region 6 (personal: white, transport: yellow, taxi: red, Governmental: blue)

Region 8: This region is standard in the Iraqi license plate, which represents the county name (IRAQ).

\section{Proposal System}

Our proposed system for License Plate Recognition System has four major stages (figure 2) which also may contain sub stages.

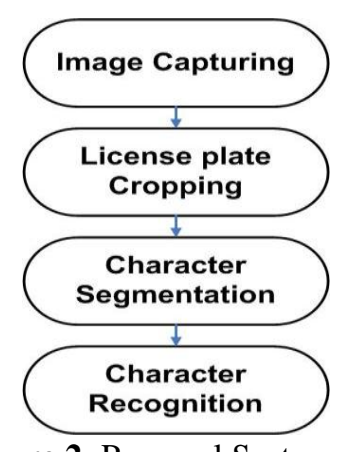

Figure 2: Proposal System

The image capturing can be done by using a digital camera with a specific distance between the gate and the vehicle that want to cross as shown in figure (3), the second stage will crop the captured image to extract only the plate frame from the whole image by using a comparison with standard plate (didn't contain any characters), then followed by multiple cropping process to detect the regions described before, the regions that contain Arabic characters will be only used in this system instead of regions that contain English characters because of the larger size of Arabic regions that will give more accurate process in the recognition stage. Next a segmentation stage will segment the selected regions.

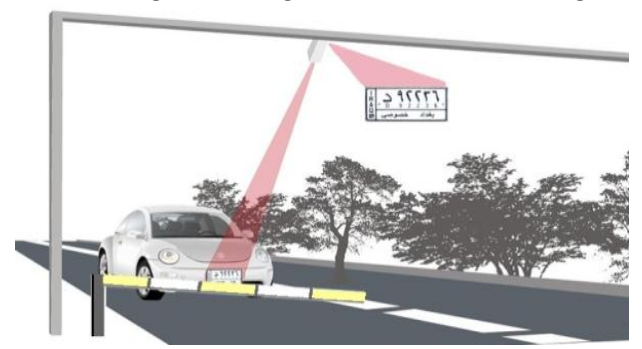

Figure 3: Capturing process

During the whole process, only 2, 3, 5 and 7 region will be used in the system, and after cropping these regions as in figure (4), the segmentation process will take a place.
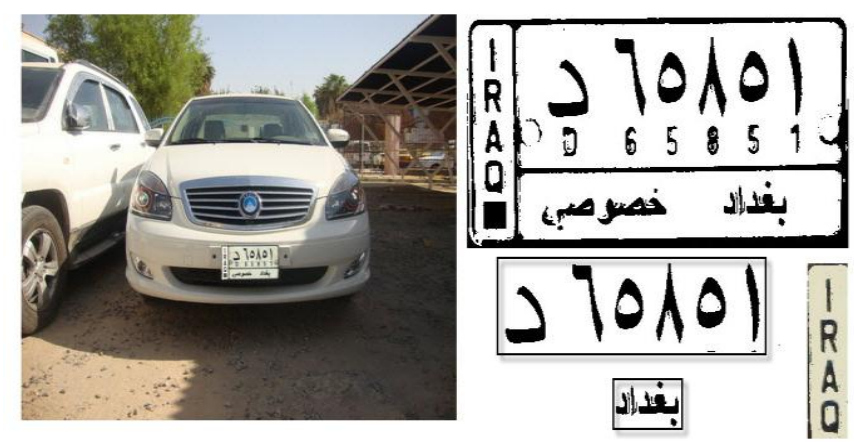

Figure 4: Licensee Plate Cropping

Segmentation for character will be performed using edge detection; which is the process of partitioning a digital image into multiple parts or sub images. Segmentation is used to simplify and/or change the representation of an image into something that is more meaningful to analyze. The algorithm of edge detection used in this system for example in number segmentation was as shown below in equation no. (1).

$$
\text { Conter(y) } \leq\left(\text { Haight } \frac{2 y}{100}\right) \text { and Conter(y-1) } \leq\left(\text { Height; } \frac{100}{100}\right) \text {... }
$$

According to the equation above if counter for white pixels in a specific column (y) is less than or equal $99 \%$ from the total number of image height and counter for white pixels in column (y-1) is equal 100\% from the total number of image height, then segmentation process will be performed.

In the Recognition stage; the letter and numbers will be compared with a database for all Indian numbers and letters to find the minimum error which will lead to the actual character, this means for example if the image is for number five; then this image will be compared with images for number zero to nine and the mismatch will be calculated to find the ratio of error, finally the minimum error is the right number. The same process will be implemented on region 5 (with database contains all governorate names), while in region 7 (car type), a different process will be applied that is finding the average color of the background for this region to recognize the type of the car, which is done by taking average value for Red, Green and Blue pixels, then according to equations the color will be defined. For example the equation (2) below will decide if the color is yellow.

Red average $\geq 100$ ard Green average $\geq 100$ ard Blue average $\leq 170 \ldots$ (2)

The system also tells us about the regions that need additional check by the user, by notifying him through check groups that are part of the graphical user interface for this system as shown in figure (5). Number checking for example shows digit need checking if there is a different between two error ratio (as described before) less than 1.5. The GUI can be modified to make the capturing automated instead of browse button. 


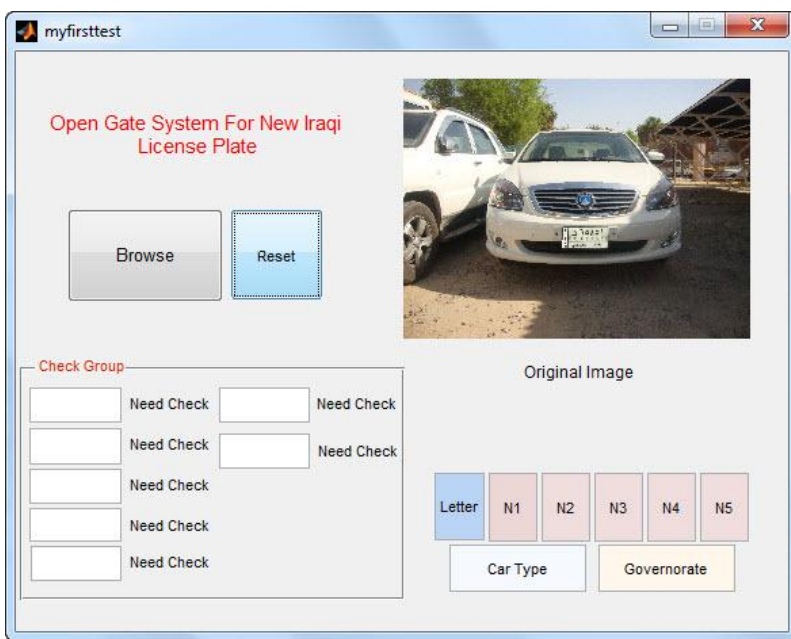

Figure 5: Graphical User Interface

\section{Results}

All experiments were done on Pentium IV $2.4 \mathrm{MHz}$ with 2048 RAM under Matlab R2012a environment. In the experiments, 30 images were employed with different image size and camera type but all have distance between camera and vehicle from 1.5 to 2 meter under different lighting conditions of the real world.

More than satisfactory result has been obtained; the success of detection rate for new Iraqi license plate is $93.33 \%$ (the $6.66 \%$ is related to fail in any region as shown in figure 6).

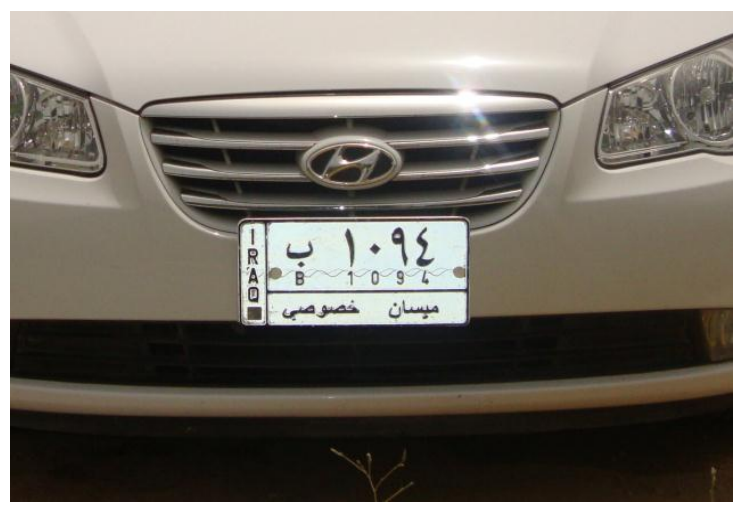

Figure 6: Graphical User Interface

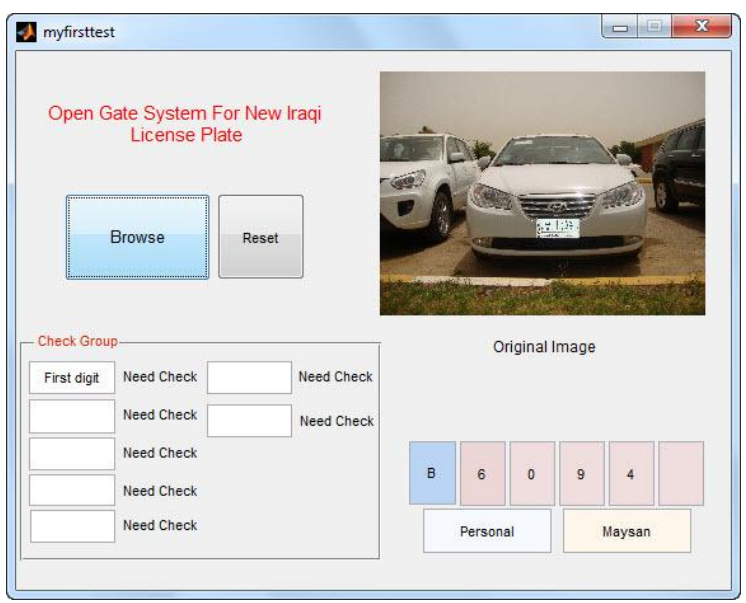

Figure 6): Graphical User Interface

In this system, most failed plates happened when vehicle images were taken in the following cases: (1) damage or dirty license plate (2) gloomy light (3) character shadow in LP due to light angle.

\section{Conclusion}

In this paper, we primarily deal with comparing images in case of cropping the plate and characters, which gave good result. The system proposed seems to be very efficient for using in open gate system especially if all cases that improve the detection exist like good lighting location, clear weather and clean LP, the time consuming can be reduced if we maintain the vehicle location. Final note is that the new Iraqi license plate contains many regions and variables that make the computerized process very difficult.

\section{References}

[1] Jian-Xia Wang et.al. 2010, The Research and Realization of Vehicle License Plate Character Segmentation and Recognition Technology, College of Information Science \& Engineering, Hebei University of Science and Technology, Wavelet Analysis and Pattern Recognition (ICWAPR), International Conference. ISBN: 978-1-4244-6530-9. DOI: 10.1109/ICWAPR.2010.5576426, pp: $101-104$

[2] Kaushik Deb, Hyun-Uk Chae and Kang-Hyun Jo 2009, Vehicle License Plate Detection Method Based on Sliding Concentric Windows and Histogram, School of Electrical Engineering and Information Systems, University of Ulsan, Ulsan, Korea, Journal of Computers, Vol 4, No 8 (2009), 771-777, Aug 2009 doi:10.4304/jcp.4.8.771-777

[3] Sudip Roy 2007, Vehicle License Plate Extraction and Recognition, Vehicle License Plate Extraction and Recognition.

[4] Wangunyu P.W, Opiyo E.T.O and Rodrigues A.J. 2009, License Plate Recognition System: Localization for Kenya, School of Computing \& Informatics, University of Nairobi, 6th Annual International Conference on Computing and ICT Research - ICCIR 10, pp: 355-366

[5] Wenjing Jia, Huaifeng Zhang, and Xiangjian He 2005, Mean Shift for Accurate Number Plate Detection, Department of Computer Systems, Faculty of Information Technology, University of Technology, Sydney, Intelligent Transportation Systems. IEEE Proceeding. pp: 566 - 571, ISBN: 0-7803-9215-9. DOI: $10.1109 /$ ITSC.2005.1520110 